\title{
Reflections The Hannan Crusaid Treatment Centre - early beginnings and lessons learnt
}

\author{
R Kaplan, MD; C Orrell, MB ChB, MSc, MMed; S D Lawn, FRCP, MD, DTM\&H, DipHIVMed; \\ L-G Bekker, MB ChB, DTM\&H, DCH, FCP (SA), PhD; R Wood, DSc (Med), FCP (SA)
}

Corresponding authors: L-G Bekker (linda-gail.bekker@hiv-research.org.za) and R Wood (robin.wood@hiv-research.org.za)

Richard Kaplan, Catherine Orrell, Stephen Lawn, Linda-Gail Bekker and Robin Wood hail from the Desmond Tutu HIV Centre, Institute of Infectious Disease and Molecular Medicine, Department of Medicine, University of Cape Town, South Africa

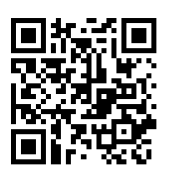

After 12 years, the Hannan Crusaid Treatment Centre (HCTC) in Gugulethu, Cape Town has screened more than 10000 women, men, adolescents and children for life-saving antiretroviral therapy (ART). While we have seen a slow but steady increase in the starting $\mathrm{CD} 4^{+}$count of new patients, the monthly inflow of new clients goes on unabated. A successful example of public-private partnership, this provincial clinic, supported by a local non-governmental organisation and initially funded by a UK-based charity, may provide a model for similar programmes within the National Health Insurance plan. Here we discuss the history and development of this programme, with a focus on lessons learnt about rolling out ART in South Africa more generally.

\section{Early beginnings}

The first community-based treatment at the Gugulethu Day Hospital was provided in 2002 by a team from the Desmond Tutu HIV Centre, literally working out of a biscuit box! Dr Catherine Orrell, Sr Felicity Cope and six counsellors opened a clinic on three days a week, bringing in their daily supplies each time in a cardboard box and having to wait for the availability of a consultation room. The first patients were extremely ill and many did not survive the first few months of ART. Shortages of medication meant that rationing decisions had to be made on a daily basis. Despite this, the team continued with enthusiasm, as every patient was known by name, many received regular home visits, and some were at times escorted to hospitals by our doctors or had ART hand-delivered to them while in hospice care.

By March 2003, the ART service was able to open full-time with the addition of Dr Kwezi Matoti (and his room in the clinic, giving a dedicated space for the service), with Sr Lulu Mtwisha heading Sizophila, a team of therapeutic counsellors who provided support for patients starting treatment. The first 150 carefully rationed treatment places were expanded to just 350 , but we endeavoured to have an open-door policy - allowing people to attend whenever they felt they needed, and never turning anyone away. While the initial focus of the treatment programme was predominantly on the provision of ART in a primary healthcare setting, over time we also established a strong research programme that enabled us to better understand the HIV epidemic and the impact of our interventions. Much of this work has gone on to inform local, national and international policies and, as elsewhere in South Africa, this demonstrates the potential synergies that academic medicine can bring to strengthening healthcare delivery. This has included insights into ART adherence, nurse-driven services to step-down ART management, earlier treatment of HIV/tuberculosis (TB) co-infection and rapid initiation of ART in pregnancy. Of note, the HCTC team helped to shape the first Western Province ART guidelines using the lessons learnt from this clinic, and these subsequently went on to inform the first national ART guidelines.

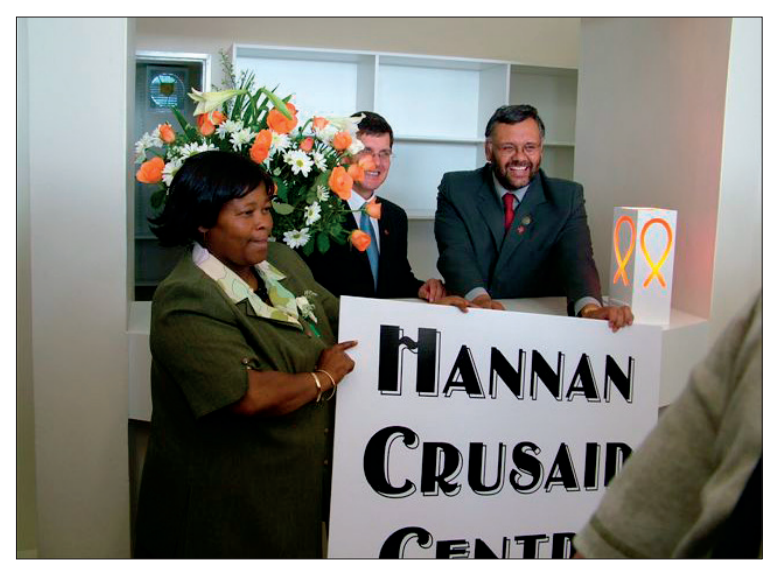

In 2001, the size of the problem made it very clear that ART would need to be delivered at primary healthcare level and money was sought to provide such a programme in the Nyanga District of Cape Town. So began a three-way partnership between the Western Cape Provincial Department of Health, led by Dr Fareed Abdullah, the Desmond Tutu HIV Foundation and CRUSAID, the UK-based AIDS Charity with a large endowment from Ms Katy Hannan.

\section{Lessons learnt \\ Adherence, loss to follow-up and virological failure}

A strong interest in adherence led us to design our programme to include three treatment-preparedness sessions and to include pill counts at each visit. Early adherence data were excellent 
and virological suppression (viral load (VL) $<50$ copies $/ \mathrm{ml}$ ) was noted in $>90 \%$ of those receiving treatment at every time point in the first 3 years. ${ }^{[1]}$ A system, initially called the 'red alert' system, was designed to identify and support those whose VL was unsuppressed (>1 000 copies $/ \mathrm{ml}$ ) at any point after starting ART. This 'red alert' system included an extra adherence-focused education session, an increased frequency of counsellor home visits, the provision of pill boxes and monthly visits to the clinic. We showed that $75 \%$ of those who experienced virological breakthrough could re-suppress their VL after this intervention. ${ }^{[2]}$ Based on counting tablet returns, adherence appears to remain high in the cohort. Adherence continues to be an important focus of the programme and, with the introduction of TB treatment in the clinic in 2011, the adherence support system was expanded to provide integrated community support for HIV/TB co-infected patients.

\section{Sizophila ('we are well') therapeutic counsellors}

In 1999, a small band of enthusiastic women who had survived HIV as participants in our early ART clinical trials stepped forward requesting to help others do the same. We taught them all we knew about ART, called them 'therapeutic counsellors' and initially asked them to work in our programmes at Somerset and Groote Schuur hospitals in Cape Town. These counsellors were the earliest accompanateurs or patient advocates and the forerunners of the community care workers we have in our primary health programme today. Elaine Dube, one of the earliest and as passionate today, went on to become involved in the very successful antenatal care Mothers2mothers programme. Initially we started with a ratio of one therapeutic counsellor to every ten patients. Community-based, they worked in the areas where they lived, knew their 'assigned' patients personally, and were highly effective for many reasons: two important ones being their deep understanding of their condition and the impact of ART in their own lives, and the intense empathy that they offered, living openly with their own infection at a time when HIV was highly stigmatised. Today they remain the cornerstone of the success of the clinic and it is well accepted that any community-based ART programme requires community-based support.

\section{HIV/TB co-infection}

Early on in the introduction of ART at HCTC, $\mathrm{TB}$ was recognised as the leading serious opportunistic disease, with more than half of patients receiving treatment for $\mathrm{TB}$ at the time that they started ART. This not only caused much suffering for affected patients, but patients with TB also had a doubled mortality risk compared with those without $\mathrm{TB}^{\left[{ }^{[3]}\right.}$

However, the problem was compounded by the difficulty in diagnosing TB in patients with HIV. We found that only $25 \%$ of patients with sputum culture-positive pulmonary TB reported having a chronic cough for longer than 2 weeks. Moreover, we described

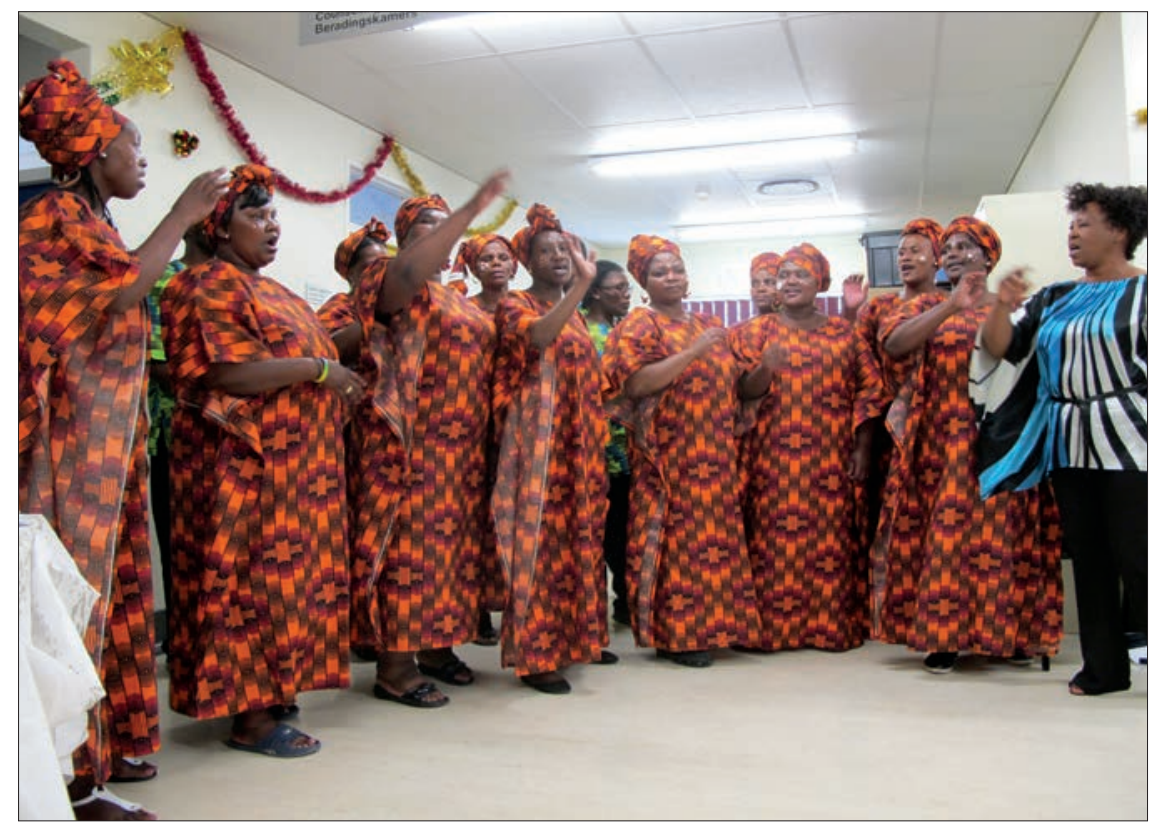

The Sizophila choir. how sputum smear microscopy and chest $\mathrm{X}$-rays are able to diagnose only a small fraction of the disease present; in turn, onefifth of unselected patients not receiving $\mathrm{TB}$ treatment at the time of first enrolment in the clinic actually have sputum culture-positive $\mathrm{TB}$, and this has led to much greater use of culture-based diagnosis.

Our studies have shown that patients who have HIV and TB face great challenges in receiving optimum and timely care when ART and TB treatment services are not integrated. In particular, ART is often unacceptably delayed. It has therefore been a huge step forward that, from 2011 onwards, patients have been able to receive TB treatment at the HCTC, and from 2012, multidrug-resistant (MDR)-TB patients have also been treated on site. We have further been able to show that increases in $\mathrm{CD}^{+}$ counts during ART are associated with major reductions in long-term TB risk.

\section{Pregnancy}

Programmes for the prevention of motherto-child transmission (PMTCT) of HIV were introduced at the Midwife Obstetric Unit (MOU) at the Gugulethu Community Health Clinic in 2001. With an antenatal care HIV prevalence rate of $29 \%$ in Gugulethu, pregnant women constituted a significant proportion of the patients who were eligible for ART. Work at the HCTC intensified to develop a 'fast track' system that would ensure a quicker route to ART commencement for pregnant and sick patients. In 2010, the Desmond Tutu HIV Foundation ran a pilot programme in partnership with the Western Cape Department of Health, demonstrating that it was safe, feasible and acceptable to initiate women whose $\mathrm{CD}^{+}$counts were $<350$ cells $/ \mu$ l on the same day as their diagnosis during the first antenatal care visit, and that by doing so transmission rates could drop to as low as $<1 \%{ }^{[4]}$ This approach is now standard in the MOU as part of growing emphasis on providing rapid ART, and ongoing care and support to HIV-positive women during pregnancy and post partum.

\section{Adolescents}

A review of the programme's outcomes confirmed what many first-world HIV programmes and other chronic disease clinical services were showing: that adolescents often experience a turbulent transitional developmental phase and may experience significant challenges in traditional ART programmes. Despite growing up in the clinic 


\section{'... it is recognised that many of the adolescents are now growing into adulthood and from 2012 the clinic developed a Health Care Transition model to facilitate the shift from adolescent to adult services.'}

as model patients, older children often lose their VL control and find it difficult to adhere and attend services as they move into the teen years. In 2005, the HCTC recognised the need to be sensitive to adolescent needs, and organised patients aged $10-22$ years into four age bands to attend clinic appointments in their specific age strata. These age-specific clinics were held in the afternoons with dedicated youth-oriented counsellors and a fun 'chill room' within the clinic. It is recognised that many of the adolescents are now growing into adulthood and from 2012 the clinic developed a Health Care Transition model to facilitate the shift from adolescent to adult services.

\section{The future}

The HCTC was one of the pioneers of community-based ART in South Africa. The early provincial and national treatment protocols, as well as a score of subsequent policies and models, have emanated from this centre. The lessons learnt have been captured in peer-reviewed publications, which have in turn influenced the treatment programmes and policies, not only in South Africa, but in many treatment programmes throughout Africa and further afield.

The Centre has become an example of what can be achieved through a public-private-academic partnership whereby, via careful application of a variety of resources - financial, logistical, expertise and innovation - in a climate of shared partnership and collaboration, much can be learnt by doing. In a nationwide, public sector health intervention such as the South African ART programme, it is critical that specific facilities and services are maintained, where critical thinking, rigorous evaluation and innovation can be applied to ensure that, in an evolving and dynamic epidemic, the best health models and programmes are being utilised.

The HCTC has, over the last 12 years, not only screened over 10000 women, men and children for the need for life-saving ART, it has in many ways reached much further to advocate for, instruct, change and improve the way in which antiretrovirals are administered on this continent and beyond. Importantly, this clinic - with its strong emphasis on patient education, patient support and mutual respect - epitomised the paradigm of the patient-centred approach that became the hallmark of HIV management. Our hope is that these lessons will continue to be learnt and written about as we enter the next decade in which we hope the flames of the HIV epidemic will be doused and we will see our patients growing older in good health.

\section{References}

1. Bekker L-G, Myer L, Orrell C, Lawn S, Wood R. Rapid scale-up of a communitybased HIV treatment service: Programme performance over 3 consecutive years in Guguletu, South Africa. S Afr Med J 2006;96(4):315-320.

2. Orrell C, Harling G, Lawn SD, et al. Conservation of first-line antiretroviral treatment regimen where therapeutic options are limited. Antivir Ther 2007;12(1):83-88.

3. Lawn SD, Myer L, Bekker L-G, Wood R. Burden of tuberculosis in an antiretroviral treatment programme in sub-Saharan Africa: Impact on treatment outcomes and implications for tuberculosis control. AIDS 2006;20(12):1605-1612. [http://dx.doi. org/10.1097/01.aids.0000238406.93249.cd]

4. Black S, Zulliger R, Myer L, et al. Safety, feasibility and efficacy of a rapid ART initiation in pregnancy pilot programme in Cape Town, South Africa. S Afr Med J 2013;103(8):557-562. [http://dx.doi.org/10.7196/SAMJ.6565]

S Afr J HIV Med 2014;15(1):35-37. DOI:10.7196/SAJHIVMED.1040 